\title{
Evolutionary genetics of lifespan and mortality rates in two populations of the seed beetle, Callosobruchus maculatus
}

\author{
CW Fox ${ }^{1}$, ML Bush $^{1}$, DA Roff ${ }^{2}$ and WG Wallin ${ }^{1}$ \\ ${ }^{1}$ Department of Entomology, S-225 Agricultural Science Center North, University of Kentucky, Lexington, KY 40546-0091, USA; \\ ${ }^{2}$ Department of Biology, University of California, Riverside, CA 92521, USA
}

\begin{abstract}
The age at which individuals die varies substantially within and between species, but we still have little understanding of why there is such variation in life expectancy. We examined sex-specific and genetic variation in adult lifespan and the shape of mortality curves both within and between two populations of the seed beetle, Callosobruchus maculatus, that differ in a suite of life history characters associated with adaptation to different host species. Mean adult lifespan and the shape of the logistic mortality curves differed substantially between males and females (males had lower initial mortality rates, but a faster increase in the rate of mortality with increasing age) and between populations (they differed in the rate of increase in mortality with age). Larger
\end{abstract}

individuals lived longer than smaller individuals, both because they had lower initial mortality rates and a slower increase in the rate of mortality with increasing age. However, differences in body size were not adequate to explain the differences in mortality between the sexes or populations. Both lifespan and mortality rates were genetically variable within populations and genetic variance/ covariance matrices for lifespan differed between the populations and sexes. This study thus demonstrated substantial genetic variation in lifespan and mortality rates within and between populations of $C$. maculatus.

Heredity (2004) 92, 170-181, advance online publication, 21 January 2004; doi:10.1038/sj.hdy.6800383

Keywords: body size; Callosobruchus maculatus; logistic mortality model; longevity; mortality rate; senescence

\section{Introduction}

The age at which individuals die varies substantially within and between species (Tatar, 2001). Although the reasons why organisms senesce and die are generally well understood (Medawar, 1952; Hamilton, 1966; Williams, 1957; Rose, 1991) the causes of the substantial variation in rates of senescence and mortality are not well understood. Some of the within-population variance in lifespan and mortality rates is genetically based (eg, Tatar and Carey, 1994a, and references therein). However, even within genetically homogeneous cohorts, individuals vary in the age at which they die. Body size is commonly observed to covary with variation in lifespan and mortality rates, although patterns are generally inconsistent across studies. In many mammals, small stature is associated with increased lifespan and reduced risk of mortality (Miller et al, 2000 and references therein). In other studies, variation in size does not covary with lifespan (eg, Nilssen, 1997) or large individuals live longer than small individuals (Møller et al, 1989). In insects, body size varies substantially across species, across populations within species, and between the sexes, potentially explaining much of the variation in lifespan that is observed in nature.

Correspondence: CW Fox, Department of Entomology, S-225 Agricultural Science Center North, University of Kentucky, Lexington, KY 405460091,USA.E-mail: cfox@uky.edu

Received 19 November 2002; accepted 12 May 2003
Part of the reason we have little understanding of what causes variation in lifespan and rates of senescence is that biologists have paid little attention to describing the variation in patterns of mortality and senescence. Instead, most studies simply document differences in mean lifespan between populations or species. However, senescence refers to intrinsic degeneration of function that produces an increase in age-specific mortality with increasing age (Tatar, 2001). Mean lifespan provides information about mortality averaged across all ages, but obscures how the patterns of mortality vary with age (Tatar and Carey, 1994b; Curtsinger et al, 1995; Pletcher et al, 2000; Tatar, 2001), and thus obscures how patterns of senescence vary. Even when comparing survival curves, which consider survival differences as a function of age, it is difficult to distinguish whether study groups differ in initial mortality rates or in the rate of change in mortality with increasing age, though the two patterns have quite different theoretical implications. Comparison of how mortality rates change with age allows the easy distinction of specific events that cause mortality (such as spikes of mortality during mating or egg laying) from differences in rates of senescence.

The mortality rate $\left(u_{x}\right)$ is a measure of the probability of dying that reflects the age-specific slope of the survivorship curve. $u_{x}$ is not bounded by 0 or 1 and is largely independent of sampling interval (Elandt-Johnson and Johnson, 1980; Allison, 1995; but see Pletcher, 1999a). Senescence is generally quantified as an increase in the mortality rate with increasing age. The rate of senescence, measured as the exponent (b) describing the 
exponential rate of increase in mortality rate $\left(u_{x}\right)$ with increasing age, has until recently been believed to vary little within species (Finch, 1990). However, recent studies have shown that rates of increase in mortality rates vary among populations or among cohorts/ genotypes within populations of a species (Tatar and Carey, 1994a; Pletcher and Curtsinger, 2000). For senescence to evolve in response to natural selection, there must be genetic variation in the shape of the mortality curve. However, few studies have examined the degree to which mortality curves vary in shape, and even fewer have demonstrated that mortality curves are genetically variable or have examined which features of the curves are genetically variable (Curtsinger et al, 1995). Most studies that have examined genetic variation in mortality curves have done so using Drosophila (but see Tatar and Carey, 1994a).

Populations of Callosobruchus maculatus vary substantially in lifespan, body size, and a suite of morphological, behavioral, and life history traits (Messina, 1990). In all populations studied, mean lifespan is higher for females than males, possibly due to a difference in the age at which mortality becomes age-dependent (Tatar and Carey, 1994b). Variation in adult lifespan within populations is heritable (Møller et al, 1989; Tatar and Carey, 1994a). Although a couple studies have shown a positive relationship between body size and adult lifespan (Møller et al, 1989; Tatar and Carey, 1994a), only Møller et al (1989) has demonstrated a significant genetic correlation between body size and lifespan.

In this study we examine two populations of C. maculatus that differ in a large suite of traits, including body size, adult lifespan, larval competitiveness, oviposition behavior, and degree of paternal investment (eg, Savalli et al, 2000). Some of these traits are known to be attributable to differences in selection associated with their host plants. For example, C. maculatus is a seed parasite. Host seed size variation between these two populations has driven differentiation in body size and larval aggressiveness; selection favors large beetles that are highly aggressive when larvae develop inside of Vigna radiata seeds, because the seeds are small and can only support the development of one or two larvae, but favors the evolution of less-aggressive larvae that are small and tolerant of scramble competition when larvae develop inside of the larger V. unguiculata (Messina, 1991; Messina and Slade, 1997). Relevant to this study is the observation that the large differences in body size between populations correspond to large differences in adult lifespan between populations (Figure 1), suggesting that host-associated selection on larval aggressiveness and body size may have driven large differences in other life history traits, including adult lifespan.

We examine sex-specific and genetic variation in adult lifespan and the shape of mortality curves both within and between populations. We then test whether (a) differences in body size between populations or (b) effects of rearing environment (host species) may be adequate to explain the differences in lifespan and mortality rates. Because we focus on the adult lifespan and patterns of mortality during the adult stage, this study largely ignores patterns of mortality during larval development (except for total larval mortality). However, differences in larval development between populations or between rearing hosts may influence patterns of adult
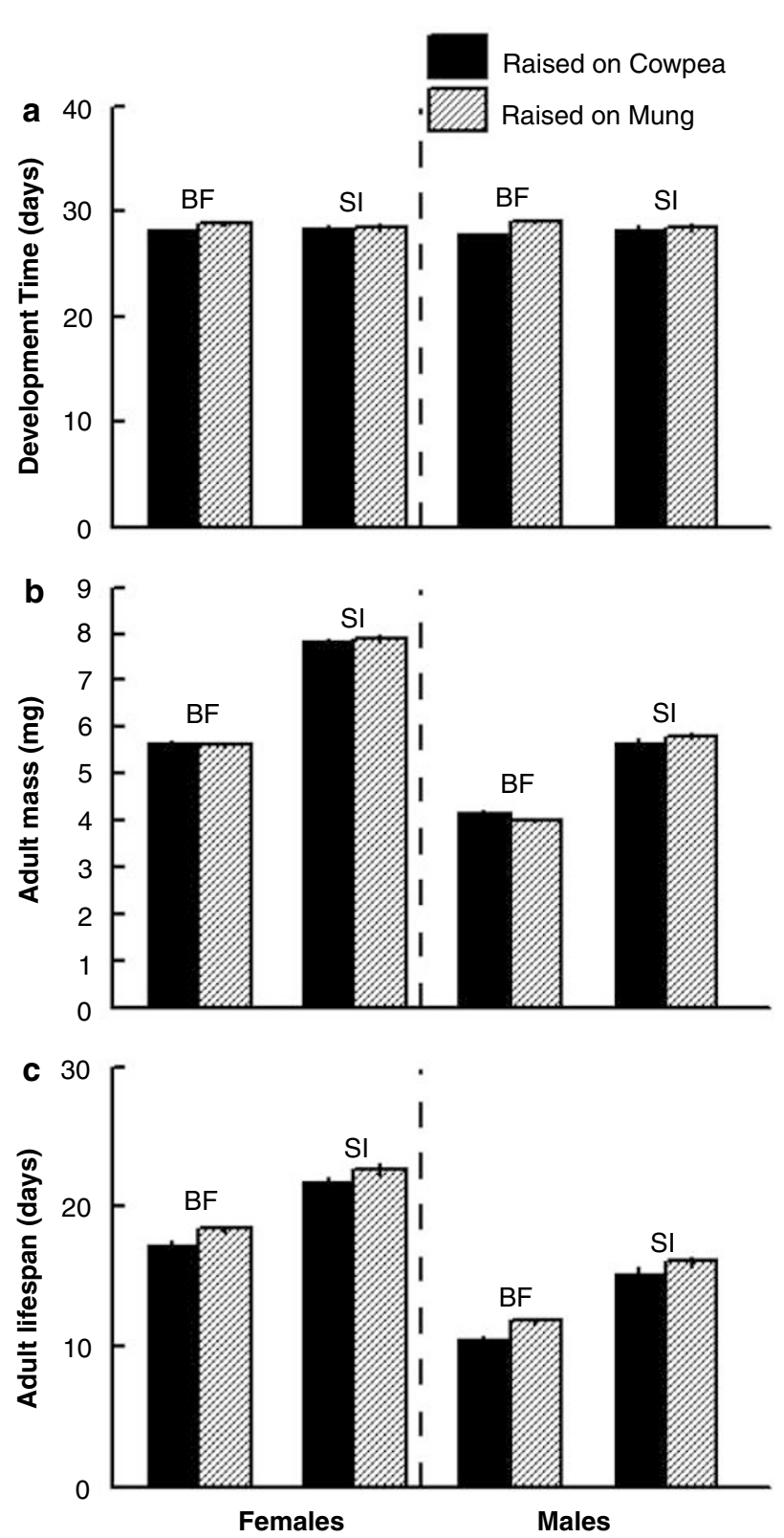

Figure 1 Population means for (a) egg-to-adult development time, (b) adult mass, and (c) adult lifespan of C. maculatus. Note that SI beetles are much larger, and live longer than BF beetles. Also note the large difference in size between males and females. Beetles were reared to adult on either seeds of cowpea ( $V$. unguiculata) or mung ( $V$. radiata).

mortality. Although we do not examine larval development in detail, we consider variation in larval egg-toadult development time as an indicator of variation in larval performance between populations and among families.

\section{Methods}

Natural history and study population

C. maculatus is a cosmopolitan pest of stored legumes (Fabaceae), particularly beans of the genus Vigna. Females cement their eggs to the surface of host seeds (Messina, 1991). Approximately 4-5 days later (at 26-28 ${ }^{\circ} \mathrm{C}$ ), 
the eggs hatch and the first instar larvae burrow through the seed coat and into the seed. Larval development and pupation are completed entirely within a single seed; larvae go through four instars plus a pupal stage inside the seeds, and then emerge as reproductively mature adults. This beetle's short generation time and ease of laboratory rearing in a seminatural storage environment make it an excellent animal for life history studies.

Having evolved to use dry seeds, and most recently having evolved in a storage environment, C. maculatus larvae develop and adults mature, mate and complete reproduction using only metabolic water and the resources acquired during larval development (ie, they are capital breeders; Messina and Slade, 1999). In some bruchids (eg, Bruchus pisorum) adult feeding (on pollen, floral or extra-floral nectarines, or leaf fungi) is common and these nutrients are used for maturation of eggs (eg, Clement, 1992). In Callosobruchus and some other storage pest bruchids access to adult resources also has a small positive effect on female fecundity and improves adult lifespan (Leroi, 1978; Shinoda and Yoshida, 1987; Fox, 1993a, b; Tatar and Carey, 1995). However, C. maculatus adults have no access to food or water in a storage environment (they cannot feed externally on seeds) and there is little evidence that they feed as adults outside of a storage environment.

We used two populations of beetles for these experiments. The South India (SI) population was collected in 1979 from infested pods of mung bean and the closely related black gram (both $V$. radiata) in Tirunelveli, India (Messina and Slade, 1997). The Burkina Faso (BF) population was collected in 1989 from infested pods of cowpea (V. unguiculata) in Ouagadougou, Burkina Faso (Messina, 1993; Messina and Slade, 1997). These two populations differ in a whole suite of traits, including body size, lifetime fecundity, patterns of egg dispersion, oviposition preference, and adult longevity (see Introduction). Both populations were maintained in laboratory growth chambers on seeds of $V$. radiata (SI) or $V$. unguiculata (BF) at $>1000$ adults per generation for $>100$ generations $(\mathrm{BF})$ or $>200$ generations $(\mathrm{SI})$ prior to this experiment.

\section{Experimental design}

We used a half-sib design to quantify genetic variation in larval developmental period, adult mass, adult lifespan, mortality rate, and the genetic correlations among these traits. To create half-sib families, virgin male beetles (44 sires from the SI population, and 51 sires from the BF population) were each mated sequentially to between two and four different virgin females. Each virgin male, collected within $12 \mathrm{~h}$ of his emergence from an isolated host seed, was isolated in a 35- $\mathrm{mm}$ Petri dish and allowed to mature for 1 day (although males are capable of mating immediately following emergence, their ejaculate is not fully formed; Fox et al, 1995). Each male was then confined in a 35-mm Petri dish with a virgin female that was $<12 \mathrm{~h}$ post-emergence, and allowed to copulate. Males were presented females sequentially, one per day, until they successfully mated with four females. Most males fertilized all four females, but a few females were not successfully fertilized resulting in fewer than four dams per sire.
Because the two populations of beetles were collected from, and maintained on, two different species of Vigna, host-species effects on mortality patterns need to be considered in any population comparison. We thus used a split brood design to raise offspring on both host species. Note that this design allows us to consider rearing host effects on lifespan but, because the parents of SI beetles were reared from a different host than the parents of $\mathrm{BF}$ beetles, our results are potentially confounded by nongenetic parental effects (Mousseau and Fox, 1998).

To collect eggs on both host species, half of the mated females from each sire were placed in a $60 \mathrm{~mm}$ Petri dish containing $\approx 40$ mung seeds ( $V$. radiata) and the other half were confined in a $60 \mathrm{~mm}$ dish with $\approx 20$ cowpea seeds ( $V$. unguiculata). Every $12 \mathrm{~h}$ the female was transferred to a new dish of seeds, alternating between the two host species such that all females laid eggs on both host species. This was continued for 3 days. We raised 24 offspring per female (12 from each host species) which, after a small amount of larval mortality, resulted in 8746 adult offspring.

Larvae were reared to adult at densities of one beetle per seed (excess eggs were scraped off), one seed per dish (to ensure that only one beetle emerged from each dish), $25^{\circ} \mathrm{C}$, L:D 15:9. Egg-to-adult mortality was very low in both populations on both hosts, but generally lower for BF larvae and lower for larvae raised on mung (BF on cowpea, 0.041; BF on mung, 0.022; SI on cowpea, 0.057; SI on mung, 0.033). Emerging beetles were collected twice daily, at $12 \mathrm{~h}$ intervals. All adults emerged solitarily into a dish and were thus virgin. A random sample of approximately two-thirds of the emerging beetles from each family (eight per family per host, chosen prior to emergence) were weighed within $12 \mathrm{~h}$ of their emergence from the seed $(N=5528)$. All beetles were subsequently transferred into a sterile $35 \mathrm{~mm}$ Petri dish and maintained at $25^{\circ} \mathrm{C}$, L:D 15:9, until death. Beetles were scored twice daily (at $12 \mathrm{~h}$ intervals) to ascertain age at death.

\section{Analyses}

A Weibull distribution was not appropriate for analyzing differences in mortality (hazard) functions $[u(t)]$ between sexes because $\log \{-\log [S(t)]\}$ vs $t$ was not linear [where $S(t)$ is survival at time $t$ ] (Parmar and Machin, 1995). Neither a Gompertz nor a Gompertz-Makeham model described mortality rates as well as a logistic mortality function of the form $u(t)=a \mathrm{e}^{b t} /\left[1+(a s / b)\left(\mathrm{e}^{b t}-1\right)\right]$, where $a$ is the initial mortality rate, $b$ is the rate of exponential increase in mortality at young ages, and $s$ describes the degree of deceleration in mortality with increasing age (Vaupel, 1990; Pletcher, 1999b). This model is similar to a Gompertz mortality model except that it incorporates a term (s) to account for the slowing of the increase of mortality rate with age (Pletcher and Curtsinger, 1998). Parameters were estimated using the maximum likelihood estimation procedure of WinModest (Pletcher, 1999b). A Cox-proportional hazards model was used to test for overall sex, host and population differences in $u(t)$ (Allison, 1995; Parmar and Machin, 1995). We used the log-likelihood-ratio test of WinModest to test whether individual parameter estimates $(a, b$ and $s)$ 
differed significantly between males and females, between rearing hosts, and between populations.

Genetic variances and covariances for adult body mass, lifespan, and egg-to-adult development time were calculated using the restricted maximum likelihood variance component estimation procedure of SAS Proc VARCOMP (Littell et al, 1991), with $V_{\mathrm{A}}$ (the additive genetic variance) $=4 V_{\mathrm{S}}$ (the among sire variance component), and the heritability $\left(h^{2}\right)=V_{\mathrm{A}} / V_{\mathrm{P}}$ (Roff, 1997). Standard errors for $V_{\mathrm{A}}$ and $h^{2}$ were calculated following Becker (1992). For comparison with these estimates, $V_{\mathrm{A}}$, $h^{2}$, and their standard errors were also calculated via jackknifing the variance component estimates across sires (within populations) using S-Plus (Venables and Ripley, 1997; Selvin, 1998). Additive genetic correlations $\left(r_{\mathrm{A}}\right)$ were calculated both between traits within sexes and between sexes. Between-trait genetic correlations were estimated using standard varcomp procedures in S-Plus (Venables and Ripley, 1997; Selvin, 1998). Standard errors for $r_{\mathrm{A}}$ estimates were calculated by jackknifing estimates across all sires within each population (Knapp et al, 1989; Windig, 1997; Fox et al, 1999). We calculated between-sex additive genetic correlations for adult lifespan and body mass from the various maximum likelihood variance components (Fry, 1992; Lynch and Walsh, 1998), as $r_{\mathrm{A}}=\sigma_{\text {sire-mixed }}^{2} / \sigma_{\text {sire-male }} \sigma_{\text {sire-female, }}$ where $\sigma_{\text {sire-mixed }}^{2}$ is the estimated sire main effect variance component from the complete mixed model analyses of variance, with progeny sex treated as factor, and $\sigma_{\text {sire-male }}$ and $\sigma_{\text {sire- }}$ female are the estimated sire main effect variance components from the two reduced models [one for each sex; data were standardized to $\operatorname{Normal}(0.1)$ to correct for differences in mean and variance between the sexes]. Only the sire (co)variance components were used to avoid possible bias due to dominance and maternal effects (Mousseau and Fox, 1998). Standard errors for $r_{\mathrm{A}}$ were estimated via the jackknife as in Fox et al (1999). The hypotheses that $r_{\mathrm{A}}<1$ was tested using a one-sample $t$-test (Knapp et al, 1989).

To compare genetic variance-covariance matrices (Gmatrices; Becker, 1992; Lynch and Walsh, 1998) between populations, sexes and hosts, we used the MANOVA method of Roff (2002). For each group, we calculated the $G$-matrix. We then deleted in turn a single sire group and calculated the pseudovalues according to the usual jackknife procedure (Potvin and Roff, 1993; Manly, 1997). The final data matrix was arranged such that the columns comprised the pseudovalues of each (co)variance and the rows the results for the deletion of a given family (so the $i$ th row $j$ th column is the pseudovalue for the $j$ th (co)variance for the sample with the $i$ th family deleted). The data set was then analyzed with MANOVA (Tabachnick and Fidell, 2001) using S-Plus (Venables and Ripley, 1997; Selvin, 1998).

\section{Results}

Population-level mortality patterns

Postemergence from the seed (ie, post-maturation), females lived on average 6.5 to 6.6 days longer than males (averaged across each population-host combination; $\mathrm{F}_{(1,8152)}=4200, P<0.001$ in the complete ANOVA; Figures 1 and 2). The mortality (hazard) functions also
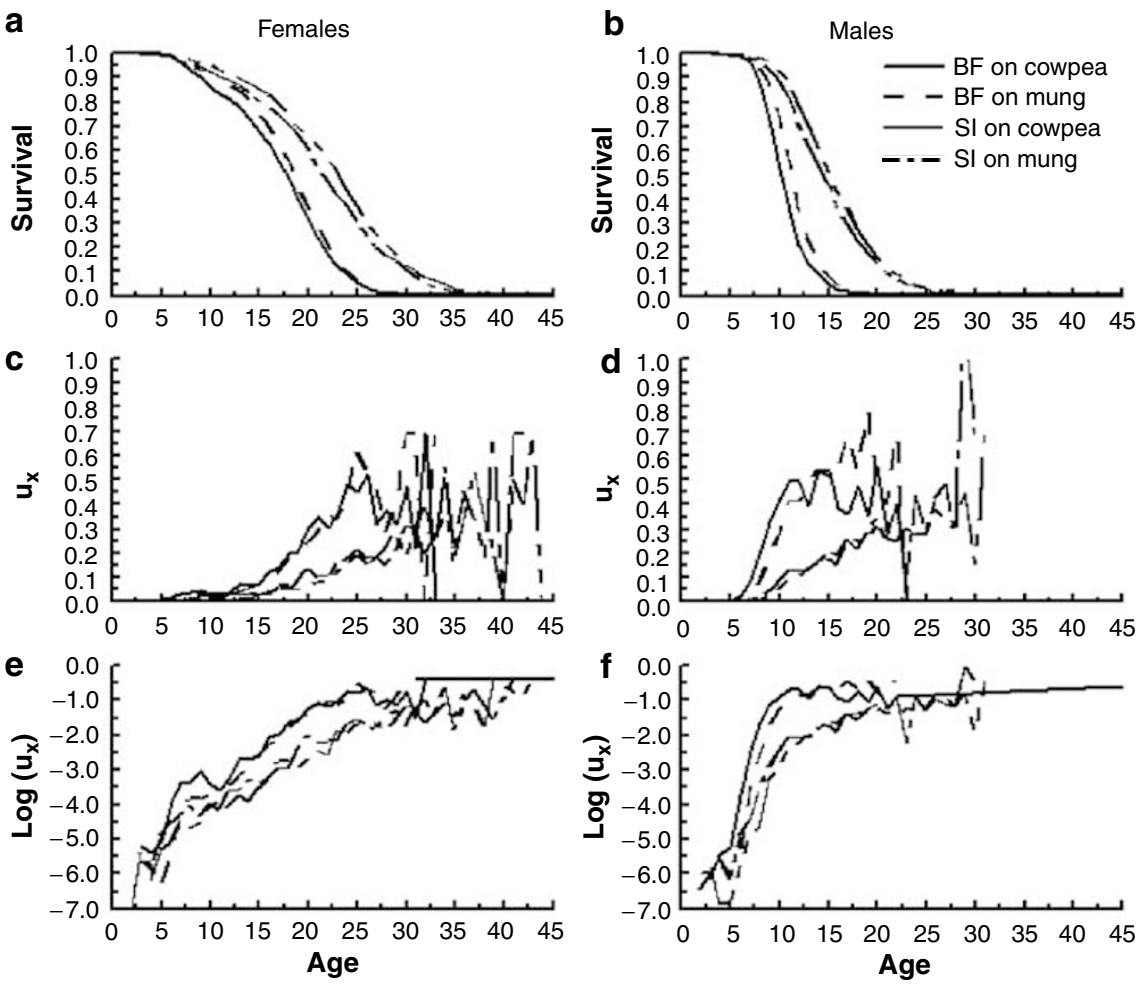

Figure 2 (a, b) Survivorship (c, d) mortality curve $[u(t)]$, and $(\mathbf{e}, \mathbf{f})$ log-transformed mortality curve, log $[u(t)]$, for male and female $C$. maculatus from the SI and BF populations. The mortality curve was best explained by the logistic mortality model, $u(t)=a \mathrm{e}^{b t} /\left[1+(a s / b)\left(\mathrm{e}^{b t}-1\right)\right]$, where $a$ is the initial mortality rate, $b$ is the rate of exponential increase in mortality at young ages, and $s$ describes the degree of deceleration in mortality with increasing age (see parameters values in Table 1). 
differed substantially between males and females for each population-host combination (Cox proportional hazards, $\chi_{(1)}^{2}>543, P<0.001$ for each; Breslow ties handling; Figure 2c-d). Mortality patterns were best described by a logistic mortality model of the form $u(t)=a \mathrm{e}^{b t} /\left[1+(a s / b)\left(\mathrm{e}^{b t}-1\right)\right]$, where $a$ is the initial mortality rate, $b$ is the rate of exponential increase in mortality at young ages, and $s$ describes the degree of deceleration in mortality with increasing age. Male and female mortality curves differed significantly in all three parameters of the mortality model $(a, b$, and $s)$ for all population-host combinations (log-likelihood-ratio test; three parameters with four pair-wise tests each; $\chi^{2}(1)>38.6, P<0.001$ for every test; WinModest) (Table 1); although females had a higher initial mortality rate than males (a) the rate of exponential increase in mortality with increasing age was substantially greater in males (ie, higher slope, b) than in females. Unlike Tatar and Carey (1994b), we did not find that males and females differed substantially in the age at which mortality switched from being age-independent to being agedependent (Figure 2e-f); in both populations, mortality began increasing exponentially between age 4 and 5 days.

Beetles reared on mung lived on average 0.8-1.2 days longer than beetles reared on cowpea (averaged across each population-sex combination; $\mathrm{F}_{(1,8152)}=88, P<0.001$ in the complete ANOVA). The mortality functions also differed substantially between beetles reared on mung and beetles reared on cowpea for each population-sex combination $\left(\chi^{2}{ }_{(1)}>10.7, P<0.002\right.$ for each). However, in pair-wise comparisons of the parameters of the mortality function for mung and cowpea reared beetles, few were

Table 1 Parameter values for the logistic mortality model, $u(t)=a \mathrm{e}^{b t} /\left[1+(a s / b)\left(\mathrm{e}^{b t}-1\right)\right]$

\begin{tabular}{cccc}
\hline & \multicolumn{2}{c}{ Parameter estimates (95\% confidence intervals) } \\
\cline { 2 - 4 } & $\mathrm{a}$ & $\mathrm{b}$ & $\mathrm{s}$ \\
\hline SI population \\
$\begin{array}{c}\text { Female offspring } \\
\text { Cowpea }\end{array}$ & 0.0020 & 0.199 & \\
& & & \\
Mung & $0.0014-0.0027)$ & $(0.179-0.222)$ & $(0.366-0.750)$ \\
& 0.0012 & 0.215 & 0.626 \\
Male offspring & $(0.0008-0.0017)$ & $(0.193-0.238)$ & $(0.457-0.857)$ \\
Cowpea & 0.0002 & 0.579 & 2.269 \\
Mung & $(0.0001-0.0004)$ & $(0.498-0.674)$ & $(1.765-2.918)$ \\
& 0.0001 & 0.609 & 2.425 \\
BF population & $(0.0000-0.0002)$ & $(0.528-0.701)$ & $(1.928-3.049)$ \\
Female offspring & & & \\
Cowpea & 0.0020 & 0.261 & 0.518 \\
Mung & $(0.0015-0.0027)$ & $(0.240-0.284)$ & $(0.393-0.682)$ \\
Male offspring & 0.0009 & 0.293 & 0.462 \\
Cowpea & $(0.0006-0.0013)$ & $(0.269-0.320)$ & $(0.335-0.638)$ \\
Mung & 0.0000 & 1.161 & 2.527 \\
& $(0.0000-0.0000)$ & $(1.045-1.290)$ & $(2.143-2.978)$ \\
& 0.0000 & 0.982 & 1.894 \\
& $(0.0000-0.0000)$ & $(0.885-1.088)$ & $(1.582-2.267)$ \\
\hline
\end{tabular}

$a$ is the initial mortality rate, $b$ is the rate of exponential increase in mortality at young ages, and $s$ describes the degree of deceleration in mortality with increasing age. significantly different between rearing hosts (Table 1; BF females: $a$ differed between hosts, $\chi^{2}(1)=13.04, P<0.001$; BF males: $b$ and $s$ differed between hosts, $\chi_{(1)}^{2}=5.20$ and 5.64, respectively, $P<0.05$ for each; all other tests, $\left.\chi_{(1)}^{2}<3.91, P>0.05\right)$.

Mean longevity differed significantly between the two beetle populations: SI beetles lived on average 3.4-4.5 days longer than $\mathrm{BF}$ beetles (averaged across each sexhost combination; $F_{(1,98)}=160, P<0.001$ in the complete ANOVA). This difference reflected a substantial difference in the shape of the mortality functions between SI and BF beetles $\left(\chi^{2}{ }_{(1)}>301, P<0.001\right.$ for each sex-host combination). SI and BF mortality curves differed significantly in the initial mortality rate $(a$; differed between populations for males only: $\chi_{(1)}^{2}>7.26, P<0.02$ for both hosts; females: $\chi^{2}{ }_{(1)}<1.49, P>0.2$ for both hosts; Table 1$)$ and rate of increase in mortality $(b$; lower slope for SI beetles; $\chi^{2}{ }_{(1)}>10.8, P<0.001$ for all four sex-host combinations; Table 1) but not for the degree of deceleration in the mortality curves $\left(s ; \chi_{(1)}^{2}<2.86\right.$, $P<0.1$ for each comparison; Table 1).

Interestingly, there was no detectable sex $\times$ population, host $\times$ population, or sex $\times$ host interaction effect on mean adult lifespan $(P>0.22$ for each in the overall ANOVA).

\section{Relationship between body size and adult lifespan}

In the SI population, larger individuals lived longer than smaller individuals, regardless of rearing host or sex. However, mass explained only a small percentage of the total variation in lifespan. In separate linear regressions for each sex-host combination, $R^{2}$ values ranged from 0.045 to 0.14 although, due to large sample sizes, the effect was always highly significant $(P<0.001$ for each host-sex combination). There was no evidence that the relationship between body size and adult lifespan differed between the sexes or between hosts (no significant mass $\times$ sex or mass $\times$ host interactions in an analysis of covariance). In the BF population, the relationship between adult size and lifespan differed between males and females; body mass affected adult lifespan of males (but $R^{2}<0.04, P<0.001$ for both rearing hosts) but there was no detectable effect of body mass on adult lifespan of females $\left(R^{2}<0.01, P>0.14\right.$ for each host). This difference between males and females was detectable in an analysis of covariance as a marginally nonsignificant mass $\times$ sex interaction $(P=0.06)$. In the complete analysis of covariance (both populations combined) there was a highly significant effect of mass $(P<0.001)$ and a highly significant population $\times$ mass interaction $(P<0.001)$ but, probably due to the small proportion of the variance explained by body size in the linear regressions, the sex $\times$ mass and population $\times$ sex $\times$ mass interactions were nonsignificant $(P>0.19$ for each). There was no evidence that the effect of mass on adult lifespan differed between host plants for either population (nonsignificant mass $\times$ host interactions).

To test which parameters of the mortality model differed between large and small individuals, we divided each population-sex-host data subset (eight combinations) into two equal parts based on size, large vs small individuals (above vs below the median). For the purpose of estimating model parameters, it was 
impractical to divide the data set into smaller size classes due to substantial loss of power for comparing parameters. For all eight population-sex-host combinations, animals in the large size class had a longer average adult lifespan than did animals in the smaller size class ( $t$-test, $P<0.05$ for all comparisons). The shape of the survival curve also differed between the body size classes for all eight pairs of data sets (Cox proportional hazards, $\chi_{(1)}^{2}>4.4, P<0.04$ for each test). However, after fitting a logistic model separately to each size class, the only parameter differences we could detect between size classes was for $s$, the rate at which mortality leveled off at increasing age (likelihood ratio test, significant for $\mathrm{BF}$ males and females reared on cowpea, and for SI females raised on cowpea, $\chi_{(1)}^{2}>4.5, P<0.04 ; P>0.05$ for all other comparisons). No other differences in parameters between size classes were significant in the individual comparisons $(P>0.05$ for each), but for seven of the eight comparisons, the estimate of $a$ was lower for larger individuals than for smaller individuals (sign test, $P<0.01$; ie, lower initial mortality rates for larger individuals), and for six of the eight, the estimate of $b$ was lower for larger individuals than for smaller individuals $(P<0.075$; ie, slower increase in the mortality rate for larger individuals).

To test the hypothesis that the body size difference between SI and BF lines (Figure 1a) is adequate to explain the difference in adult lifespan between populations (Figure 1b), we first removed the linear effect of body size from the analysis (using linear regression) and tested whether residuals from the body mass vs adult lifespan regression differed between populations (separate regression analyses for each sex and rearing host). The lifespan difference between populations was still highly significant $\left(\mathrm{F}_{(1,5143)}=31.92, P<0.001\right)$, indicating that the lifespan difference between populations cannot be fully explained by the linear relationships between body mass and adult lifespan within each sex.

We tested whether body size could explain the differences in lifespan between the two sexes using the same type of residual analysis (the residuals were calculated separately for each population and rearing host). The lifespan difference between the sexes was still highly significant $\left(\mathrm{F}_{(1,5143)}=166.10, P<0.001\right)$, indicating that the lifespan difference between males and females cannot be fully explained by the linear relationships between body mass and adult lifespan within each sex.

\section{Genetic variation and covariation}

Within populations, the shape of the mortality curves varied significantly among sire families (pooling dams; Cox proportional hazards, $\chi_{(1)}^{2}>23.4, P<0.001$ for both populations) and among dam (full-sib) families $\left(\chi^{2}(1)>24.5, P<0.001\right)$, demonstrating that there is genetic variation in the shape of mortality curves within both populations (note that this analysis is potentially confounded by maternal effects but, because beetles were raised individually rather than in groups the variation in the shape of the mortality curve among families is not confounded by covariance among siblings due to a common-rearing environment). Unfortunately, we were unable to test for variation in the specific parameters $a, b$, and $s$ due to limited family sizes within each sire.
The MANOVA analysis indicated that the G-matrices (the genetic variance-covariance matrices) differed significantly between populations (Wilks' $\lambda=0.969$, $\left.\mathrm{F}_{(6,370)}=3.93, \quad P=0.009\right)$ and between the sexes (Wilks' $\left.\lambda=0.979, F_{(6,370)}=2.67, P=0.047\right)$. We did not detect a significant difference between the two rearing host species (Wilks' $\lambda=0.995, \mathrm{~F}_{(6,370)}=0.61, P=0.60$ ) nor any population $\times$ sex, population $\times$ host, or sex $\times$ host interaction $(P>0.47$ for each).

Estimates of the heritability $\left(h^{2}\right)$ of adult lifespan varied between 0.251 and 0.702 (Table 2). In SI beetles, $h^{2}$ was higher in males than in females (Table 2), although the difference was only significant for non-jackknifed estimates for beetles reared on cowpea. This gender difference in $h^{2}$ was due to a substantial difference between the sexes in the amount of environmental variance (estimates of $V_{\mathrm{E}}$ ranged between 34 and 37 for females, but only between 7 and 9 for males). The higher $V_{\mathrm{E}}$ for females resulted in much higher $V_{\mathrm{P}}$ and thus lower $h^{2}$. $V_{\mathrm{E}}$ was likewise higher for females than for males for $\mathrm{BF}$ beetles (Table 2). However, $V_{\mathrm{A}}$ was also substantially higher in females than in males (Table 2) such that the proportion of $V_{\mathrm{P}}$ explained by $V_{\mathrm{A}}\left(h^{2}\right)$ was actually the same for both sexes in the BF population. Because the means for adult lifespan differed substantially between males and females, the differences in $V_{\mathrm{A}}$ and $V_{\mathrm{E}}$ could be artifacts of a difference in scale between the sexes (Houle, 1992; Messina, 1993). Thus, we calculated the coefficient of environmental variation for lifespan and found that the large difference in $V_{\mathrm{E}}$ between males and females disappeared in the $\mathrm{BF}$ population (no difference in $\mathrm{CV}_{\mathrm{E}}$ between males and females), but that $\mathrm{CV}_{\mathrm{E}}$ was still higher for females in the SI population $(\approx 45 \%$ higher $)$

Estimates for the heritability $\left(h^{2}\right)$ of body mass varied between 0.367 and 0.646 , but did not differ between hosts, sexes or populations (Table 2 ; $t$-tests, $P>0.05$ ). This range of heritabilities is similar to the range of heritabilities of body size found for other populations of C. maculatus (Møller et al, 1989; Messina, 1993; Fox, 1994; Tatar and Carey, 1994a; Guntrip et al, 1997) and for other seed beetles (Fox, 1998). There was no detectable genetic variation in egg-to-adult development time. Overall estimates of $V_{\mathrm{A}}$ and $h^{2}$ of development time tended to be higher for SI beetles than for BF beetles, but none of the estimates differed significantly from zero, and none differed between hosts, sexes, or populations (Table 2).

As demonstrated with the regression analyses above, body size was phenotypically correlated with lifespan for SI males and females, and for BF males, but not for BF females (Table 3), and these correlations differed between populations and between the sexes, but not between rearing hosts. However, none of the significantly positive phenotypic correlations between adult mass and adult lifespan reflected underlying positive genetic correlations; instead, three of the eight estimated genetic correlations were actually negative, and none differed significantly from zero (Table 3). Lifespan remained heritable after the removal of body mass for all eight population-host-sex combinations $(\mathrm{F}>1.63, P<0.02)$ except $\mathrm{BF}$ males raised from cowpea $\left(\mathrm{F}_{(50,157)}=1.26, P=0.14\right)$ which was the lowest estimate of $h^{2}$ prior to removing the effect of body mass (Table 2). 
Table 2 Phenotypic, genetic and environmental variances $( \pm S E M)$ in adult body mass, adult lifespan (lifespan postemergence from the host seed), and egg-to-adult development time

\begin{tabular}{|c|c|c|c|c|c|}
\hline & \multicolumn{5}{|c|}{ Variance components } \\
\hline & $\begin{array}{l}\text { Varcomp additive } \\
\text { genetic variance }\left(\mathrm{V}_{A}\right)\end{array}$ & $\begin{array}{l}\text { Jackknifed additive } \\
\text { genetic variance }\left(\mathrm{V}_{A}\right)\end{array}$ & $\begin{array}{l}\text { Environmental } \\
\text { variance }^{b}\left(\mathrm{~V}_{E}\right)\end{array}$ & $\begin{array}{c}\text { Varcomp narrow-sense } \\
\text { heritability }\left(\mathrm{h}^{2}\right)\end{array}$ & $\begin{array}{c}\text { Jackknifed narrow-sense } \\
\text { heritability }\left(\mathrm{h}^{2}\right)\end{array}$ \\
\hline \multicolumn{6}{|l|}{ Adult body mass } \\
\hline \multicolumn{6}{|c|}{ SI population } \\
\hline \multicolumn{6}{|c|}{ Female offspring } \\
\hline Cowpea & $0.335+0.141$ & $0.328+0.167$ & 0.579 & $0.367+0.145$ & $0.348+0.173$ \\
\hline Mung & $0.530 \pm 0.194$ & $0.495 \pm 0.182$ & 0.291 & $0.646 \pm 0.209$ & $0.603 \pm 0.209$ \\
\hline \multicolumn{6}{|c|}{ Male offspring } \\
\hline Cowpea & $0.252 \pm 0.126$ & $0.218 \pm 0.084$ & 0.391 & $0.392 \pm 0.184$ & $0.288 \pm 0.141$ \\
\hline Mung & $0.185 \pm 0.094$ & $0.476 \pm 0.141$ & 0.295 & $0.385 \pm 0.185$ & $0.423 \pm 0.182$ \\
\hline \multicolumn{6}{|c|}{ BF population } \\
\hline \multicolumn{6}{|c|}{ Female offspring } \\
\hline Cowpea & $0.223 \pm 0.091$ & $0.171 \pm 0.085$ & 0.380 & $0.370 \pm 0.143$ & $0.371 \pm 0.134$ \\
\hline Mung & $0.451 \pm 0.148$ & $0.206 \pm 0.087$ & 0.315 & $0.589 \pm 0.173$ & $0.608 \pm 0.167$ \\
\hline \multicolumn{6}{|c|}{ Male offspring } \\
\hline Cowpea & $0.167 \pm 0.060$ & $0.194 \pm 0.083$ & 0.171 & $0.493 \pm 0.164$ & $0.568 \pm 0.217$ \\
\hline Mung & $0.168 \pm 0.063$ & $0.168 \pm 0.058$ & 0.214 & $0.440 \pm 0.151$ & $0.430 \pm 0.140$ \\
\hline \multicolumn{6}{|l|}{ Adult lifespan } \\
\hline \multicolumn{6}{|c|}{ SI population } \\
\hline \multicolumn{6}{|c|}{ Female offspring } \\
\hline Cowpea & $13.98+7.09$ & $16.49+7.73$ & 36.52 & $0.277+0.135$ & $0.300+0.134$ \\
\hline Mung & $14.86 \pm 7.01$ & $21.08 \pm 8.63$ & 34.42 & $0.301 \pm 0.136$ & $0.392 \pm 0.150$ \\
\hline \multicolumn{6}{|c|}{ Male offspring } \\
\hline Cowpea & $13.39 \pm 4.53$ & $8.95 \pm 4.69$ & 7.15 & $0.652 \pm 0.192$ & $0.699 \pm 0.309$ \\
\hline Mung & $11.76 \pm 3.91$ & $7.81 \pm 3.09$ & 8.65 & $0.576 \pm 0.170$ & $0.702 \pm 0.254$ \\
\hline \multicolumn{6}{|c|}{ BF population } \\
\hline \multicolumn{6}{|c|}{ Female offspring } \\
\hline Cowpea & $11.30 \pm 3.95$ & $14.01 \pm 6.85$ & 19.61 & $0.366 \pm 0.119$ & $0.283 \pm 0.144$ \\
\hline Mung & $8.03 \pm 2.72$ & $14.71 \pm 6.45$ & 16.10 & $0.333 \pm 0.106$ & $0.315 \pm 0.126$ \\
\hline \multicolumn{6}{|c|}{ Male offspring } \\
\hline Cowpea & $2.67 \pm 1.20$ & $3.28 \pm 1.21$ & 7.42 & $0.265 \pm 0.114$ & $0.251 \pm 0.207$ \\
\hline Mung & $3.33 \pm 1.05$ & $3.21 \pm 1.46$ & 2.91 & $0.534 \pm 0.151$ & $0.509 \pm 0.218$ \\
\hline \multicolumn{6}{|c|}{ Egg-to-adult development time } \\
\hline \multicolumn{6}{|c|}{ SI population } \\
\hline \multicolumn{6}{|c|}{ Female offspring } \\
\hline Cowpea & $1.43 \pm 1.39$ & $2.54 \pm 1.75$ & 9.76 & $0.128 \pm 0.123$ & $0.172 \pm 0.113$ \\
\hline Mung & $8.49 \pm 5.61$ & $20.83 \pm 19.63$ & 22.91 & $0.270 \pm 0.174$ & $0.538 \pm 0.442$ \\
\hline \multicolumn{6}{|c|}{ Male offspring } \\
\hline Cowpea & $5.67 \pm 3.59$ & $1.03 \pm 0.72$ & 11.57 & $0.329 \pm 0.200$ & $0.446 \pm 0.410$ \\
\hline Mung & $8.54 \pm 6.93$ & $-0.34 \pm 0.67^{c}$ & 28.91 & $0.228 \pm 0.181$ & $0.190 \pm 0.264$ \\
\hline \multicolumn{6}{|c|}{ BF population } \\
\hline \multicolumn{6}{|c|}{ Female offspring } \\
\hline Cowpea & $0.21 \pm 0.62$ & $9.76 \pm 8.54$ & 4.84 & $0.042 \pm 0.122$ & $0.239 \pm 0.198$ \\
\hline Mung & $0^{\mathrm{a}}$ & $9.04 \pm 11.32$ & 5.33 & $\overline{0^{\mathrm{a}}}$ & $-0.071 \pm 0.160$ \\
\hline \multicolumn{6}{|c|}{ Male offspring } \\
\hline Cowpea & $0^{\mathrm{a}}$ & $0.23 \pm 0.65$ & 5.77 & $0^{\mathrm{a}}$ & $0.059 \pm 0.171$ \\
\hline Mung & $0.03 \pm 0.59$ & $0.99 \pm 0.95$ & 6.14 & $0.004 \pm 0.096$ & $0.199 \pm 0.192$ \\
\hline
\end{tabular}

Genetic variances were calculated using the restricted maximum likelihood variance component estimation procedure of SAS Proc VARCOMP (Littell et al, 1991). Standard errors for $h^{2}$ calculated following Becker (1992). See Fox (1998) and Fox et al (1999) for other examples of these procedures.

${ }^{\text {a} E s t i m a t e d ~ a m o n g-s i r e ~ v a r i a n c e ~ c o m p o n e n t ~ w a s ~} 0$, resulting in a $0 V_{\mathrm{A}}$ and $0 \mathrm{~h}^{2}$.

${ }^{b}$ Estimated assuming dominance, epistasis and maternal effects are 0 . Neither maternal effects nor dominance explained more than $5 \%$ of the variance for any analysis except development time, for which maternal effects were often large, calculated from the restricted maximum likelihood variance component estimation procedure of SAS Proc VARCOMP.

${ }^{\mathrm{c}}$ Although genetic variances cannot be negative, the estimates from the jackknife pseudovalues can be negative.

Phenotypically, body size was positively correlated with development time (all estimates are positive and five of the eight estimates are significantly different from zero; Table 3). However, because some estimates of $V_{\mathrm{A}}$ for development time were zero (Table 2), genetic correlations between development time and other traits could not be calculated for some of the sex-host combinations. In general, the jackknife pseudovalues varied so substantially that estimates of $r_{\mathrm{A}}$ between development time and both body mass and adult 
Table 3 Phenotypic and genetic correlations ( \pm SEM) between adult body mass, adult lifespan, and egg-to-adult development time. Estimates and standard errors were calculated by jackknifing the varcomp estimates

\begin{tabular}{|c|c|c|}
\hline & $\begin{array}{l}\text { Phenotypic } \\
\text { correlations }\end{array}$ & $\begin{array}{l}\text { Additive genetic } \\
\text { correlations }\end{array}$ \\
\hline \multicolumn{3}{|c|}{ Adult body mass - lifespan } \\
\hline \multicolumn{3}{|c|}{ BF population } \\
\hline \multicolumn{3}{|c|}{ Female offspring } \\
\hline Cowpea & $0.049 \pm 0.041$ & $0.119 \pm 0.333$ \\
\hline Mung & $0.055 \pm 0.052$ & $0.042 \pm 0.389$ \\
\hline \multicolumn{3}{|c|}{ Male offspring } \\
\hline Cowpea & $0.161 \pm 0.067$ & $0.410 \pm 0.256$ \\
\hline Mung & $0.118 \pm 0.052$ & $-0.031 \pm 0.318$ \\
\hline \multicolumn{3}{|c|}{ SI population } \\
\hline \multicolumn{3}{|c|}{ Female offspring } \\
\hline Cowpea & $0.232 \pm 0.039$ & $-0.252 \pm 0.393$ \\
\hline Mung & $0.213 \pm 0.062$ & $0.092 \pm 0.254$ \\
\hline \multicolumn{3}{|c|}{ Male offspring } \\
\hline Cowpea & $0.371 \pm 0.042$ & $0.159 \pm 0.496$ \\
\hline Mung & $0.225 \pm 0.047$ & $-0.078 \pm 0.285$ \\
\hline \multicolumn{3}{|c|}{ Adult body mass - development time } \\
\hline \multicolumn{3}{|c|}{ BF population } \\
\hline \multicolumn{3}{|c|}{ Female offspring } \\
\hline Cowpea & $-0.169 \pm 0.046$ & $-0.264 \pm 0.404$ \\
\hline Mung & $-0.185 \pm 0.049$ & $0^{\mathrm{a}}$ \\
\hline \multicolumn{3}{|c|}{ Male offspring } \\
\hline Cowpea & $-0.068 \pm 0.064$ & $-1.817 \pm 1.885$ \\
\hline Mung & $-0.275 \pm 0.043$ & $-0.568 \pm 0.698$ \\
\hline \multicolumn{3}{|c|}{ SI population } \\
\hline \multicolumn{3}{|c|}{ Female offspring } \\
\hline Cowpea & $-0.094 \pm 0.046$ & $0.107 \pm 0.436$ \\
\hline \multirow{2}{*}{\multicolumn{3}{|c|}{ Male offspring }} \\
\hline & & \\
\hline Cowpea & $-0.077 \pm 0.042$ & $-0.303 \pm 0.750$ \\
\hline Mung & $-0.089 \pm 0.073$ & $-0.078 \pm 0.285$ \\
\hline
\end{tabular}

${ }^{\mathrm{a}} 0$ in the denominator, resulting in an undefined $r_{\mathrm{A}}$.

lifespan ranged between -1.8 and 2.8 (Table 3 ). We thus found them generally uninterpretable and deleted development time from our G-matrix comparison.

In the SI population, adult lifespan of males was highly positively genetically correlated with the adult lifespan of females (additive genetic correlation between males and females, $r_{\mathrm{A}}=0.782 \pm 0.095$ for beetles reared on cowpea and $0.836 \pm 0.059$ for mung); that is, sires that produced long-lived sons also produced long-lived daughters, and vice versa. The cross-sex genetic correlation was significantly lower in the BF population $\left(r_{\mathrm{A}}=0.486 \pm 0.303\right.$ for cowpea and $0.325 \pm 0.198$ for mung). Interestingly, the cross-sex genetic correlations for body size showed the reverse pattern, higher in the $\mathrm{BF}$ population $\left(r_{\mathrm{A}}=0.895 \pm 0.047\right.$ for cowpea and $0.912 \pm 0.038$ for mung) than the SI population $\left(r_{\mathrm{A}}=0.279 \pm 0.336\right.$ for cowpea and $0.141 \pm 0.267$ for mung).

\section{Discussion}

\section{Mortality patterns}

In many animals, females live longer than males (Hazzard, 1986; Smith and Warner, 1989). In both populations of $C$. maculatus examined here, females lived longer as adults than did males. This sex difference was not due to higher initial mortality rates of males females actually had higher initial mortality rates than did males ( $a$ in Table 1). Instead, the rate of increase in the mortality rate with increasing age was much greater for males than for females. For a different population of C. maculatus (from California, USA), Tatar and Carey found that mortality was largely age-independent in males until age 5 days, at which there is an abrupt transition to age-dependency (Figure 1 in Tatar and Carey, 1994b). They found that this abrupt change-point occurred in females much later (age 11-12 days), explaining much of the sex difference in mean adult lifespan. In our two populations, we did not find that males and females differed substantially in the age at which mortality switched from being age-independent to age-dependent; in both populations, mortality began increasing exponentially between age 4 and 5 days. The sex difference in C. maculatus lifespan in the BF and SI populations was due largely to a difference in the rate of exponential increase in mortality with increasing age rather than the change-point from age-independent to dependent mortality. This difference between studies highlights that although it is a common pattern among animals that males live shorter lives than females, the mechanistic explanation for these lifespan differences must necessarily differ among species, and even among populations within species (Tatar et al, 1997).

Mean lifespan also differed substantially between the two populations; SI beetles lived longer than BF, regardless of sex. This difference was due to a greater rate of increase in mortality rate with increasing age $(b)$ in SI than BF beetles. We initially expected that body size would explain much of the difference in lifespan between populations, and between males and females, because the body size difference between populations and the sexes correspond in magnitude to the mean lifespan differences (Figure 1). Since Callosobruchus mate and reproduce using primarily larval-acquired resources (Messina and Slade, 1999), the amount of resources acquired during larval development (which affects larval and adult size) should have a large effect on adult longevity. However, like most studies with Drosophila and other insects (eg, Tatar and Carey, 1994a; Rodriguez et al, 1999; Norry and Loeschcke, 2002; but see Møller et al, 1989) our study found that the relationship between size and lifespan to be very weak and the underlying genetic correlation between size and lifespan was indistinguishable from zero suggesting little cause and effect relationship between the two. This is consistent with the results of Tatar and Carey (1994a) who found weak phenotypic correlations but no genetic correlation between body size and adult lifespan in another population of C. maculatus. However, Møller et al (1989) found a highly positive additive genetic correlation body mass and adult lifespan $\left(r_{\mathrm{A}}=0.76 \pm 0.16\right)$ and results of Messina and Fry (2003) suggest a slightly positive genetic correlation between lifespan and body size. Interestingly, in another seed beetle, Stator limbatus, males are larger than females (by 5-10\%) but have significantly shorter lifespans, indicating that size is not the primary explanation for sex-based differences in mortality in that species (Fox et al, 2003). We thus require some explanation other than size differences to explain the sex- and population-based differences in lifespan. 
Traditionally, mortality patterns have been described using a Gompertz model (Finch, 1990), which includes terms for initial mortality (an intercept) and an exponent that describes the rate of exponential increase in mortality (the slope of the $\log [u(x)]$ vs age plot). However, recent studies have found that the rate of increase in mortality slows with increasing age and that a Gompertz model is not adequate for describing these mortality patterns and that the parameters of a Gompertz model can be misleading when there is significant slowing with age (eg, Promislow et al, 1996). The parameter $s$ in the logistic mortality model describes the rate at which the exponential increase in mortality decelerates with increasing age (Pletcher, 1999a,b). We observe, for both sexes in both populations of C. maculatus, that the rate of increase of mortality gradually slows until mortality rates reach a plateau. Similar slowing of age-specific mortality rates and mortality plateaus are observed in other large-scale mortality studies (reviewed by Pletcher and Curtsinger, 1998), but are generally undetectable in smaller experiments due to the very small number of animals left alive when the plateau is reached. Such leveling off appears to be a real property of animal mortality patterns and is not the result of high adult density (Khazaeli et al, 1995, 1996), contamination of experimental lines (Brooks et al, 1994), differential aging of experimental cohorts or an artifact of using inbred lines (Hughes and Charlesworth, 1994). For example, our study maintained and tracked adults individually until death and used a single cohort of a genetically highly variable outbred line. Although our study was large enough to detect a mortality plateau in both sexes of both populations (8746 individuals in total), and to test for differences among populations and sexes in the value of $s$, we were unable to measure the exact value of the plateau or test for differences in the value of the plateau because few animals were still alive this late in the experiment. However, visually we can estimate the plateau at approximately $u(x)=0.40$ (an instantaneous risk of death of 0.40 ) and similar between sexes and populations. This is substantially higher than the plateaus observed in Tatar and Carey's (1994b) experiments with a California population of Callosobruchus (plateau at $\approx 0.20 \%$ ) and higher than for most other organisms studied (Pletcher and Curtsinger, 1998).

The reason mortality patterns reach a plateau is unknown. Redundancy models and reliability theory, which assume that organisms are composed of multiple subsystems and that the organism can survive as long as a subset of these subsystems are functioning (which depends on subsystem reliability) predict that the rate of increase in mortality rates should decelerate with increasing age (ie, $s \neq 0$ ) and can, in some situations, generate mortality plateaus (Gavrilov and Gavrilova, 2001). Alternatively, the plateau may be an artifact of heterogeneity among individuals within populations age-specific mortality rates may always increase exponentially but vary among cohorts or genotypes within a population (ie, different Gompertz curves for different subpopulations; Pletcher and Curtsinger, 1998; Partridge and Mangel, 1999). Genotypes or cohorts with either steeper individual mortality curves or higher initial mortality rates will die sooner, leaving less-frail individuals in the population at advanced ages (Vaupel and Yashin, 1985; Vaupel, 1990; Horiuchi and Wilmorth,
1998). Although this hypothesis has not been well tested, substantial heterogeneity in mortality rates does exist in most well-studied animal populations (eg, Pletcher and Curtsinger, 2000), and was observed in our study (variation among families). However, theoretical explorations indicate that heterogeneity well beyond the amount generally observed for quantitative traits need be present in a population to generate a plateau (Vaupel and Carey, 1993; Pletcher and Curtsinger, 1998; Drapeau et al, 2000; but see Service, 2000). Alternatively, antagonistic pleiotropy, in which some mutations have beneficial effects on survival at one age and negative effects at another age, can generate mortality plateaus (Mueller and Rose, 1996; but see Pletcher and Curtsinger, 1998; Wachter, 1999).

Recently, Fox and Moya-Laraño (2003) demonstrate that deceleration of the mortality rate observed at old ages, and the resulting mortality plateau, is a statistical property of any time-to-event trait that is affected by multiple genes or environmental factors. They develop a null model in which the expected mortality curve for a polygenic trait can be estimated from the observed mean and variance in lifespan. We compared the mortality curves for SI and BF beetles to the predicted curves from the null model. This comparison demonstrated that males of both populations of $C$. maculatus deviate from the null model differently than do females. Males show a significantly lower baseline mortality rate (lower intercept, a), but a higher rate of increase in the mortality rate (higher slope, b) than predicted by the null model. In contrast, females show a significantly higher baseline mortality rate and lower rate of increase than predicted by the null model. This difference between males was observed in both populations and for both rearing hosts. Interestingly, data for one other population of C. maculatus (Tatar and Carey, 1994b) and another species of seed beetle (Stator limbatus; Fox et al, 2003) also show that males have a rate of increase in the mortality rate than predicted by the null model, although in both of those analyses female mortality curves did not differ from those predicted by the null model (Fox and MoyaLaraño, 2003).

\section{Evolutionary genetics of body size and lifespan}

Adult lifespan was heritable in C. maculatus, with the heritability ranging between 0.265 and 0.652 depending on the sex, host and population. These values are comparable to estimates found in other studies of C. maculatus (Møller et al, 1989), other species of seed beetles (Nomura and Yonezawa, 1990; Tuciæ et al, 1991; Tanaka, 1993), other species of insects (Roff, 1992), and humans (Christiansen and Vaupel, 1996) but generally higher than observed in Drosophila (eg, Curtsinger et al, 1995; Promislow et al, 1996; but see Hughes, 1995). However, for senescence to evolve in response to natural selection, there must be genetic variation in the shape of the mortality curve, and not just in the mean lifespan. We found significant genetic variation within populations in the shape of mortality curves. Unfortunately, Callosobruchus family sizes are not large enough for us to accurately estimate the parameters of the logistic mortality curve separately for each family (which requires hundreds of individuals per family; Tatar and Carey, 1994b; Promislow et al, 1996), so we were unable to test which parameters of the mortality curve were genetically 
variable. Because of the requirement for very large sample sizes, studies of genetic variation in mortality curves are most easily performed on organisms where diverse genetic lines can be examined, allowing large numbers of genetically identical or very similar animals to be raised per line (but see Tatar and Carey, 1994a). Thus, the genetics of mortality curves have been examined almost exclusively in Drosophila, in which genetic variation in one or all three parameters has been detectable (Curtsinger et al, 1992; Hughes, 1995; Promislow et al, 1996; but see Pletcher et al, 1998).

Although differences between the sexes for genetic (co)variance matrices have been reported for a number of traits in a few well-studied organisms, such as Drosophila and mice (eg, Eisen and Legates, 1966; Cowley et al, 1986; Badyaev and Hill, 2000), very few have examined how genetic variances and heritabilities for lifespan differ between the sexes. We found significant differences between males and females in $V_{\mathrm{A}}$, but only in one population, while $h^{2}$ differed only in the other population. This discrepancy was due to a large difference between the sexes in $V_{\mathrm{E}}$ in both populations. Part of this difference in $V_{\mathrm{E}}$ was an artifact of large differences in mean lifespan between males and females. However, after correcting for the difference in mean lifespan (by calculating coefficients of variance, CV) females still showed inflated environmental variance relative to males (higher $\left(\mathrm{CV}_{\mathrm{E}}\right.$ ) in the SI population. Few studies have examined how $V_{\mathrm{E}}$ differs between the sexes, but those that calculate $\mathrm{V}_{\mathrm{E}}$ for both sexes rarely find large differences (Cowley et al, 1986), although Promislow et al (1996) found a pattern in Drosophila similar to what we observed (although they did not observe the inflated $V_{\mathrm{E}}$ across all age classes). It is unclear why we should observe inflated $V_{\mathrm{E}}$ in females. Because all beetles in our study were unmated virgins, the high $V_{\mathrm{E}}$ in females may reflect variation in their ability to resorb eggs or to shutdown egg production in the absence of mates or oviposition substrates (Wilson and Hill, 1989; Messina and Slade, 1999).

Alternatively, the high $V_{\mathrm{E}}$ we observe may reflect greater dominance or maternal effects affecting lifespan of females than lifespan of males. Unfortunately, our half-sib design does not allow us to disentangle dominance genetic variation or maternal effects variation from environmental variation. Previous studies have shown that life history traits (such as lifespan) are often affected by substantial amounts of dominance variation, much more so than morphological traits (such as body size; Roff, 1997). We know of no studies demonstrating that dominance variation should differ between males and females, but numerous studies have demonstrated that different genes affect male and female traits (including longevity) providing the opportunity for different genic and allelic interactions, such as different degrees of dominance, in the two sexes (see discussion below).

In both populations, the adult lifespan of females was positively genetically correlated to the adult lifespan of males, but the cross-sex genetic correlations were significantly less than 1.0. The cross-sex genetic correlation also differed substantially between the populations, being almost twice as large in the SI population $\left(r_{\mathrm{A}}\right.$ between 0.782 and 0.836$)$ as the $\mathrm{BF}$ population $\left(r_{\mathrm{A}}\right.$ between 0.325 and 0.486 ). This suggests that the genetic basis for the sexual dimorphism in lifespan differs between the populations - largely the same genes influence lifespan of males and females in the SI population, while either different genes affect lifespan of males and females in the BF population, or the genes have different effects on lifespan in males and females. This result requires more thorough examination. However, it is not surprising that different genes affect male and female lifespan in C. maculatus since recent QTL studies have demonstrated that different loci affect male and female lifespan in Drosophila (Vieira et al, 2000; Mackay, 2001). Interestingly, in another experiment we found that the mode of inheritance (type and degree of allelic and genic interactions) of the population differences in lifespan differed between males and females the difference in male lifespan between SI and BF beetles were influenced by a large maternal effect whereas the differences in female lifespan between SI and BF beetles were substantially influenced by dominance and epistasis (C Fox, in preparation).

\section{$\mathrm{SI}$ and BF populations of $C$. maculatus}

The SI and BF populations of C. maculatus differ substantially in adult size (SI are larger), adult longevity (SI live longer), larval competitiveness (SI are contest competitors, BF are scramble competitors), egg size, oviposition preference (SI prefer to oviposit on mung seeds, BF prefer cowpea), egg dispersion (SI disperse eggs more uniformly) and degree of paternal investment (greater male investment into reproduction in BF males) (Messina and Slade, 1997; Savalli et al, 2000). Some of these life history differences are directly attributable to seed size differences between mung and cowpea, which selects for differences in female egg-laying strategies, larval competitive ability, and body size. We expected that the large difference in body size, which is clearly under selection associated with seed size and larval competition, would explain the difference in adult lifespan and mortality patterns. Our data suggest that body size is not adequate to explain host-associated differences in adult lifespan, nor the sex difference in adult lifespan. Thus, at this time we are unable to identify the reason why mortality patterns differ between populations, but ongoing experiments are testing whether differences in the patterns of inheritance can explain the population and sex differences and, because populations differ in their patterns of allocation to reproduction, we are testing whether differences between sexes and populations in the cost of reproduction explains the observed differences in mortality patterns. These experiments are on-going and will be reported in a later manuscript.

\section{Acknowledgements}

We thank M Fulkerson for help during the experiment, and thank A Amarillo, ME Czesak, F Messina, J MoyaLaraño, C Rauter, and C Stillwell for comments on earlier drafts of this manuscript. F Messina kindly provided access to his beetle populations. S Pletcher kindly provided free access to his software (WinModest) for fitting and comparing mortality curves. Funding for this research was provided by NSF DEB-9996371 and DEB0110754 to $\mathrm{C}$ Fox. Funding for M Bush was provided by 
the Kentucky Young Scientist Summer Research Program at the University of Kentucky.

\section{References}

Allison PD (1995). Survival Analysis using the SAS System: A Practical Guide. SAS Institute Inc.: Cary, NC.

Becker WA (1992). Manual of Quantitative Genetics. Academic Enterprises: Pullman, Washington.

Brooks A, Lithgow GJ, Johnson TE (1994). Mortality rates in a genetically heterogeneous population of Caenorhabditis elegans. Science 263: 668-671.

Badyaev AV, Hill GE (2000). The evolution of sexual dimorphism in the house finch I. Population divergence in morphological covariance structure. Evolution 54: 1784-1794.

Christiansen K, Vaupel JW (1996). Determinants of longevity: genetic, environmental and medical factors. I Internal Med 240: 333-341.

Clement SL (1992). On the function of pea flower feeding by Bruchus pisorum. Entomol Exp Appl 63: 115-121.

Cowley DE, Atchley WR, Rutledge JJ (1986). Quantitative genetics of Drosophila melanogaster. I. Sexual dimorphism in genetic parameters for wing traits. Genetics 114: 549-566.

Curtsinger JW, Fukui HH, Khazaeli AA, Kirscher A, Pletcher SD, Promislow DEL et al. (1995). Genetic variation and aging. Annu Rev Gen 29: 553-575.

Curtsinger JW, Fukui HH, Townsend DR, Vaupel JW (1992). Demography of genotypes: failure of the limited lifespan paradigm in Drosophila melanogaster. Science 258: 461-463.

Drapeau MD, Gass EK, Simison MD, Mueller LD, Rose MR (2000). Testing the heterogenity theory of late-life mortality plateaus by using cohorts of Drosophila melanogaster. Exp Gerontol 35: 71-84.

Eisen EJ, Legates JE (1966). Genotype-sex interaction and the genetic correlation between the sexes for body weight in Mus musculus. Genetics 54: 611-623.

Elandt-Johnson R, Johnson NL (1980). Survival Models and Data Analysis. Wiley: New York, NY.

Finch CE (1990). Longevity, Senescence and the Genome. University of Chicago Press: Chicago, IL, USA.

Fox CW (1993a). Multiple mating, lifetime fecundity and female mortality of the bruchid beetle Callosobruchus maculatus (Coleoptera: Bruchidae). Funct Ecol 7: 203-208.

Fox CW (1993b). The influence of maternal age and mating frequency on egg size and offspring performance in Callosobruchus maculatus (Coleoptera: Bruchidae). Oecologia 96: 139-146.

Fox CW (1994). Maternal and genetic influences on egg size and larval performance in a seed beetle: multigenerational transmission of a maternal effect? Heredity 73: 509-517.

Fox CW (1998). Genetic and maternal influences on body size and development time in the seed beetle, Stator limbatus (Coleoptera: Bruchidae). Ann Entomol Soc Am 91: 128-134.

Fox CW, Czesak ME, Mousseau TA, Roff DA (1999). The evolutionary genetics of an adaptive maternal effect: egg size plasticity in a seed beetle. Evolution 53: 552-560.

Fox CW, Dublin L, Pollitt SJ (2003). Gender differences in mortality rates in two seed beetle species. Funct Ecol 17: 619-626.

Fox CW, Hickman DL, Raleigh EL, Mousseau TA (1995). Paternal investment in a seed beetle (Coleoptera: Bruchidae): influence of male size, age, and mating history. Ann Entomol Soc Am 88: 100-103.

Fox CW, Moya-Laraño J (2003). Late-life mortality plateaus: a null model for comparing patterns of mortality. Evol Ecol Res 5: 999-1009.

Fry JD (1992). The mixed-model analysis of variance applied to quantitative genetics - biological meaning of the parameters. Evolution 46: 540-550.

Gavrilov LA, Gavrilova NS (2001). The reliability theory of aging and senescence. J Theor Biol 213: 527-545.
Guntrip J, Sibly RM, Holloway GJ (1997). The effect of novel environment and sex on the additive genetic variation and covariation in and between emergence body weight and development period in the cowpea weevil, Callosobruchus maculatus (Coleoptera, Bruchidae). Heredity 78: 158-165.

Hamilton WD (1966). The moulding of senescence by natural selection. J Theor Biol 12: 12-45.

Hazzard WR (1986). Biological basis of the sex differential. J Am Geriatrics Soc 34: 455-471.

Horiuchi S, Wilmorth JR (1998). Deceleration in the age pattern of mortality at older ages. Demography 35: 391-412.

Houle D (1992). Comparing evolvability and variability of quantitative traits. Genetics 130: 195-204.

Hughes KA (1995). The evolutionary genetics of male lifehistory characters in Drosophila melanogaster. Evolution 49: 521-537.

Hughes KA, Charlesworth B (1994). A genetic analysis of senescence in Drosophila. Nature 367: 64-66.

Khazaeli AA, Xiu L, Curtsinger JW (1995). Effect of adult cohort density on age-specific mortality in Drosophila melanogaster. J Gerontol 50: 262-269.

Khazaeli AA, Xiu L, Curtsinger JW (1996). Effect of density on age-specific mortality in Drosophila: a density supplementation experiment. Genetica 98: 21-32.

Knapp SJ, Bridges WC, Yang M (1989). Nonparametric confidence estimators for heritability and expected selection response. Genetics 121: 891-898.

Leroi B (1978). Feeding of adults of Acanthoscelides obtectus Say (Coleoptera, Bruchidae) - influence upon the longevity and ovarian production of virgin individuals. Ann Zool Ecol Anim 10: 559-567.

Littell RC, Freund RJ, Spector PC (1991). SAS System for Linear Models, 3rd edn. SAS Institute Inc.: Cary, NC.

Lynch M, Walsh B (1998). Genetics and analysis of quantitative traits. Sinauer Associates, Inc.: Sunderland, MA USA.

Mackay TF (2001). Quantitative trait loci in Drosophila. Nat Rev Gen 2: 11-20.

Manly BFJ (1997). Randomization, Bootstrap and Monte Carlo Methods in Biology. Chapman \& Hall: New York.

Medawar PB (1952). An Unsolved Problem in Biology. H. K. Lewis: London.

Messina FJ (1990). Alternative life-histories in Callosobruchus maculatus: environmental and genetic bases. In: Fujii K, Gatehouse AMR, Johnson CD, Mitchell R, Yoshida T (eds) Bruchids and Legumes: Economics, Ecology, and Coevolution. Kluwer Academic Publishers: the Netherlands. pp 303-315.

Messina FJ (1991). Life-history variation in a seed beetle: adult egg-laying vs larval competitive ability. Oecologia 85: 447-455.

Messina FJ (1993). Heritability and 'evolvability' of fitness components in Callosobruchus maculatus. Heredity 71: 623-629.

Messina FJ, Fry JD (2003). Environment-dependent reversal of a life history trade-off in the seed beetle Callosobruchus maculatus. J Evol Biol 16: 501-509.

Messina FJ, Slade AF (1997). Inheritance of host-plant choice in the seed beetle Callosobruchus maculatus (Coleoptera: Bruchidae). Ann Entomol Soc Am 90: 848-855.

Messina FJ, Slade AF (1999). Expression of a life-history tradeoff in a seed beetle depends on environmental context. Physiol Entomol 24: 358-363.

Miller RA, Chrisp C, Atchley W (2000). Differential longevity in mouse stocks selected for early life growth trajectory. J Gerontol Ser A - Biol Sci Med Sci 55: B455-B461.

Møller H, Smith RH, Sibly RM (1989). Evolutionary demography of a bruchid beetle. I. Quantitative genetical analysis of the female life history. Funct Ecol 3: 673-681.

Mousseau TA, Fox CW (1998). Maternal Effects as Adaptations. Oxford University Press: New York, NY.

Mueller LD, Rose MR (1996). Evolutionary theory predicts latelife mortality plateaus. Proc Natl Acad Sci 93: 15249-15253.

Nilssen AC (1997). Factors affecting size, longevity and fecundity in the reindeer oestrid flies Hypoderma tarandi 
(L.) and Cephenemyia trompe (Modeer). Ecol Entomol 22: 294-304.

Nomura T, Yonezawa K (1990). Genetic correlations among life history characters of adult females in the azuki bean weevil, Callosobruchus chinensis (L.) (Coleoptera: Bruchidae). Appl Entomol Zool 25: 423-430.

Norry FM, Loeschcke V (2002). Temperature-induced shifts in associations of longevity with body size in Drosophila melanogaster. Evolution 56: 299-306.

Parmar MKB, Machin D (1995). Survival Analysis: A Practical Approach. John Wiley \& Sons: Chichester, UK.

Partridge L, Mangel M (1999). Messages from mortality: the evolution of death rates in the old. Trends Ecol Evol 14 438-442.

Pletcher SD (1999a). Model fitting and hypothesis testing for age-specific mortality data. J Evol Biol 12: 430-439.

Pletcher SD (1999b). WinModest, Ver. 1.0.2. Max Planck Institute for Demographic Research: Rostock, Germany.

Pletcher SD, Curtsinger JW (1998). Mortality plateaus and the evolution of senescence: why are old-age mortality rates so low? Evolution 52: 454-464.

Pletcher SD, Curtsinger JW (2000). The influence of environmentally induced heterogeneity on age-specific genetic variance for mortality rates. Genet Res 75: 321-329.

Pletcher SD, Houle D, Curtsinger JW (1998). Age-specific properties of spontaneous mutations affecting mortality in Drosophila melanogaster. Genetics 148: 287-303.

Pletcher SD, Khazaeli AA, Curtsinger JW (2000). Why do life spans differ? Partitioning mean longevity differences in terms of age-specific mortality parameters. J Gerontol Ser A - Biol Sci Med Sci 55: B381-B389.

Potvin C, Roff DA (1993). Distribution-free and robust statistical methods: viable alternatives to parametric statistics? Ecology 74: 1617-1628.

Promislow DEL, Tatar M, Khazaeli AA, Curtsinger JW (1996). Age-specific patterns of genetic variance in Drosophila melanogaster. I. Mortality. Genetics 143: 839-848.

Rodriguez C, Fanara JJ, Hasson E (1999). Inversion polymorphism, longevity, and body size in a natural population of Drosophila buzzatii. Evolution 53: 612-620.

Roff DA (1992). The Evolution of Life Histories. Chapman \& Hall: New York, USA.

Roff DA (1997). Evolutionary Quantitative Genetics. Chapman \& Hall: New York, NY.

Roff DA (2002). Comparing G matrices: a MANOVA approach. Evolution 56: 1286-1291.

Rose MR (1991). Evolutionary Biology of Aging. Oxford University Press: New York.

Savalli UM, Czesak ME, Fox CW (2000). Paternal investment in the seed beetle, Callosobruchus maculatus (Coleoptera: Bruchidae): Variation among populations. Ann Entomol Soc Am 93: $1173-1178$

Selvin S (1998). Modern Applied Biostatistical Analysis using S-Plus. Oxford University Press: New York.
Service PM (2000). Heterogeneity in individual mortality risk and its importance for evolutionary studies of senescence. Am Nat 156: 1-13.

Shinoda K, Yoshida T (1987). Effect of fungal feeding on longevity and fecundity of the azuki-bean weevil, Callosobruchus chinensis (L.) (Coleoptera: Bruchidae), in the azukibean field. Appl Entomol Zool 22: 465-473.

Smith DWEm, Warner HR (1989). Does genotypic sex have a direct effect on longevity? Exp Gerontol 24: 277-288.

Tabachnick BG, Fidell LS (2001). Using Multivariate Statistics. Allyn and Bacon: Boston.

Tanaka Y (1993). A genetic mechanism for the evolution of senescence in Callosobruchus chinensis (the azuki bean weevil). Heredity 70: 318-321.

Tatar M (2001). Senescence. In: Fox CW, Roff DA, Fairbairn DJ (eds) Evolutionary Ecology: Concepts and Case Studies. Oxford University Press: New York, USA. pp 128-141.

Tatar M, Carey JR (1994a). Genetics of mortality in the bean beetle Callosobruchus maculatus. Evolution 48: 1371-1376.

Tatar M, Carey JR (1994b). Sex mortality differentials in the bean beetle: Reframing the question. Am Nat 144: 165-175.

Tatar M, Carey JR (1995). Nutrition mediates reproductive trade-offs with age-specific mortality in the beetle Callosobruchus maculatus. Ecology 7: 2066-2073.

Tatar M, Gray DW, Carey JR (1997). Altitudinal variation for senescence in Melanoplus grasshoppers. Oecologia 111: 357-364.

Tuciæ N, Miloševiæ M, Gliksman I, Milanoviæ D, Aleksiæ I (1991). The effects of larval density on genetic variation and covariation among life-history traits in the bean weevil (Acanthoscelides obtectus Say). Funct Ecol 5: 525-534.

Vaupel JW (1990). Relative risks: frailty models of life history data. Theor Popul Biol 37: 220-234.

Vaupel JW, Carey JR (1993). Compositional interpretations of medfly mortality. Science 260: 1666-1667.

Vaupel JW, Yashin AI (1985). Heterogeneity's ruses: some surprising effects of selection on population dynamics. Am Stat 39: 176-195.

Venables WN, Ripley BD (1997). Modern Applied Statistics with SPlus, 2nd edn. Springer-Verlag: New York.

Vieira C, Pasyukova EG, Zeng ZB, Hackett JB, Lyman RF, Mackay TF (2000). Genotype-environment interaction for quantitative trait loci affecting life span in Drosophila melanogaster. Genetics 154: 213-227.

Wachter KW 1999. Evolutionary demographic models for mortality plateaus. Proc Natl Aacd Sci USA 96: 10544-10547.

Williams GC (1957). Pleiotropy, natural selection, and the evolution of senescence. Evolution 11: 398-411.

Wilson K, Hill L (1989). Factors affecting egg maturation in the bean weevil Callosobruchus maculatus. Physiol Entomol 14: $115-126$.

Windig JJ (1997). The calculation and significance testing of genetic correlations across environments. I Evol Biol 10 853-874. 